\title{
SOME LINEAR AND NONLINEAR EQUATIONS OF HIGHER ORDER
}

\author{
Nadezda A. Chuesheva*, Aleksandr V. Chueshev
}

Kemerovo State University, Krasnaya Str. 6, Kemerovo, 650043 Russian Federation

Received April 26, 2016;

* e-mail: chuesheva@ngs.ru

Accepted in revised form June 18, 2016;

Published June 18, 2016

Abstract: In this article we consider boundary value problems for some linear and nonlinear differential equations with partial derivatives of the sixth, fifth, fourth and third orders. We write out conditions on equation coefficients for which existence and uniqueness of solutions from Sobolev's space occur. If these conditions on equation coefficients are not valid, then there are given examples when solution is not unique, or is not unstable, or does not belong to Sobolev's space from existence and uniqueness theorem even for analytical coefficients and analytical right side of differential equation. After S.P. Novikov's fundamental study in 1974 the interest to the nonlinear Korteweg-de Vries equation, Kadomtsev-Petviashvili equation and other nonlinear equations significantly grew. In this study of such equations we used methods of algebraic geometry integration and expansion method. In these studies exact solutions of special nonlinear equations series in partial derivatives play a big role. Solvability of similar equations was also studied in articles of A.I. Kozhanov, N.A. Larkin and other authors. The aim of this article is to find some exact solutions for special series partial differential equations. Solution graphs of such problems for linear equations and for the Kortewegde Vries, Burgers-Korteweg-deVries, and Kadomtsev-Petviashvili equations are constructed.

Keywords: linear and nonlinear equations, Korteweg-de Vries equation, boundary conditions, non-uniqueness of solution, instability of solution, graphs of solutions

\section{INTRODUCTION}

Equations of higher order were studied in papers of V.N. Vragov [1], G.V. Devidenko, S.V. Uspensky [2], E.I. Egorov, S.G. Pyatkov, S.G. Popov [3], and A.V. Chueshev [5]. Formulations of boundary problems for the fourth-order equations were studied in the paper of A.I. Kozhanov [4].

Let us consider an equation in a domain $D=\left\{(x, t) \in \mathbf{R}^{2}, \quad x \in\left(0, x_{0}\right), t \in\left(0, t_{0}\right)\right\}$.

$$
\begin{gathered}
a_{1}(x, t) u_{t t t}+a_{2}(x, t) u_{t t}+a_{3}(x, t) u_{t}+a_{4}(x, t) u_{x x x x x x}+ \\
+a_{5}(x, t) u_{x x x x x}+a_{6}(x, t) u_{x x x x}+a_{7}(x, t) u_{x x x}+a_{8}(x, t) u_{x x}+ \\
+a_{9}(x, t) u_{x}+a_{10}(x, t) u=f(x, t), a_{j}(x, t) \in \mathbf{C}^{6}(\bar{D}) \\
j=1,2, \ldots, 10 .
\end{gathered}
$$

The aim of this article is to prove the existence and uniqueness theorem of weak solution, construct exact solutions and explicit examples of ill-posed problems for the Eq. (1). Such equations can be used to regularize mixed type equations of second order as it was suggested in [1]. Also, the Korteweg-de Vries linearized equation refers to (1). A review of studies on this subject in the modern theory of partial differential equations is given in this article.

Calculation of exact and numerical solutions, a progressing wave in particular, and linear equations solutions plays a big role in the soliton theory. Equations of mathematical models of physical phenomena that occur in engineering industry, chemistry, biology, mechanics and physics are usually described by nonlinear equations. Thus, it is very important to find exact solutions of nonlinear partial differential equation which can help to understand mechanisms of these physical models.

\section{Third-order differential equation (1) with respect to $t$}

Problem 1. Let us consider the following equation in $D$

$$
\begin{gathered}
u_{t t t}+a_{1} u_{t t}+a_{2} u_{t}+u_{x x x x x x}+a_{3} u_{x x x x x}+a_{4} u_{x x x x}+ \\
+a_{5} u_{x x x}+a_{6} u_{x x}+a_{7} u_{x}+a_{8} u=f(x, t), a_{j} \in \mathbf{R}, \\
j=1,2, \ldots, 8,
\end{gathered}
$$

with boundary conditions

$$
\begin{gathered}
\left.u\right|_{t=0}=\left.u_{t}\right|_{t=0, t=t_{0}}=0, \\
\left.u\right|_{x=0, x=x_{0}}=\left.u_{x x}\right|_{x=0, x=x_{0}}=\left.u_{x x x x}\right|_{x=0, x=x_{0}}=0 .
\end{gathered}
$$

Theorem 1. Let it be that $f(x, t) \in L_{2}(D)$ and equation coefficients (2) satisfy conditions:
1) $a_{3}=a_{5}=a_{7}=0$;
2) $a_{1}<0, a_{4}<0, a_{6}>0$;
3) $3+2 a_{1}\left(t_{0}-t\right) \geq \delta_{1}>0$;
4) $-2 a_{8}\left(t_{0}-t\right)-a_{2} \geq \delta_{2}>0$.

Then a solution for (2), (3), and (4) exists and is unique for the space $H^{3,2}(D)$.

Please cite this article in press as: Chuesheva N.A., Chueshev A. V. Some linear and nonlinear equations of higher order. Science Evolution, 2016, vol. 1, no. 1, pp. 46-52.

Copyright (c) 2016, KemSU. This is an open access article distributed under the terms of the Creative Commons Attribution 4.0 International License (http://creativecommons.org/licenses/by/4.0/), allowing third parties to copy and redistribute the material in any medium or format and to remix, transform, and build upon the material for any purpose, even commercially, provided the original work is properly cited and states its license. This article is published with open access at http://science-evolution.ru/. 
The space $H^{3,2}(D)$ is a closure of the space of infinitely differentiable functions satisfying boundary conditions (3) and (4) with respect to the norm

$$
\|u\|_{H^{3,2}(D)}=\left(\int_{D}\left(u^{2}+u_{x}^{2}+u_{t}^{2}+u_{x x}^{2}+u_{t t}^{2}+u_{x x x}^{2}\right) d D\right)^{\frac{1}{2}} \text {. }
$$

The theorem is proved as in [6].

Problem 2. If conditions of the theorem for coefficients of this equation are not satisfied, then a solution of this problem is not unique. Let the following conditions be satisfied for coefficients of the Eq. (3):

1) $a_{3}-a_{5}+a_{7}=0$

2) $a_{1}=-1$;

3) $a_{2}=1$;

4) $-1+a_{4}-a_{6}+a_{8}=0$.

A non-zero solution for the boundary problems (3) and (4) if $x_{0}=\pi, t_{0}=\frac{2 \pi}{\sqrt{3}}$ for the Eq. (2) is the function

$$
u(x, t)=\sin x\left(\sqrt{3}+e^{\frac{t}{2}}\left(\sin \frac{\sqrt{3}}{2} t-\sqrt{3} \cos \frac{\sqrt{3}}{2} t\right)\right) .
$$

Fig. 1 shows a graph of this solution in the 3-dimensional space.

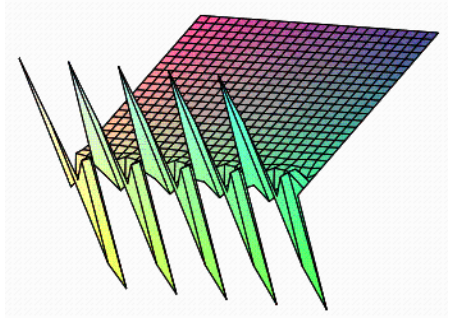

Fig. 1

Problem 3. Let us consider in the domain $D=\left\{(x, t) \in \mathbf{R}^{2}, \quad x \in(0, \pi), t \in(0, \infty)\right\}$ the following equation

$$
u_{t t t}-u_{t t}-u_{t}+u_{x x x x x x}+u_{x x x x}-u_{x x}=0
$$

with initial conditions

$$
\left.u\right|_{t=0}=\frac{\sin n x}{n^{6}},\left.u_{t}\right|_{t=0}=\frac{\sin n x}{n^{4}},\left.u_{t t}\right|_{t=0}=\frac{\sin n x}{n^{2}}
$$

and boundary conditions (4). An unstable solution for this problem is the function

$$
u(x, t)=\frac{e^{n^{2} t} \sin n x}{n^{6}} .
$$

Problem 4. Let us consider in $D$ an equation

$$
u_{t t t}-u_{t t}-u_{t}+u_{x x x x x x}-u_{x x x x}+2 u_{x x}-u=f(x, t),
$$

with boundary conditions (3) and (4).

Theorem 2. Let it be that $f(x, t) \in L_{2}(D)$. Let an inequality $3-2 t_{0} \geq 0$ be hold. Then a solution of the problems (3) and (4) for the Eq. (5) from $H^{3,2}(D)$ exists and is unique.

Theorem is proved as in [6]

Problem 5. Let us consider the Eq. (5) with the right side $f(x, t)=0$ and initial conditions $(n \in \mathbf{N})$ in $D=\left\{(x, t) \in \mathbf{R}^{2}, x>0, t>0\right\}$

$$
\left.u\right|_{t=0}=\left.u_{t}\right|_{t=0}=\left.u_{t t}\right|_{t=0}=e^{\frac{-x}{n}} .
$$

An unstable solution for this problem is the function $u(x, t)=e^{\frac{t-x}{n}}$.

An unstable solution for the Cauchy problem with the respect to $x$ in the same domain $D$

$\left.u\right|_{x=0}=\left.u_{x}\right|_{x=0}=\left.u_{x x}\right|_{x=0}=\left.u_{x x x}\right|_{x=0}=\left.u_{x x x x}\right|_{x=0}=\left.u_{x x x x x}\right|_{x=0}=e^{\frac{-t}{n}}$

of the Eq. (5) is the function $u(x, t)=e^{\frac{-t+x}{n}}$.

Problem 6. Let it be that $t_{0}=\frac{2 \pi}{\sqrt{3}}$. Note that the equation $\quad y=e^{-x}+\sqrt{2} \sin \left(x-\frac{\pi}{4}\right)=0 \quad$ has a denumerable number of solutions on the ray $[0, \infty)$ (Fig. 2). Let us consider only two solutions of this equation 0 and $x_{0}$, where $x_{0} \in\left(\pi+\frac{\pi}{4}, \pi+\frac{\pi}{2}\right)$.

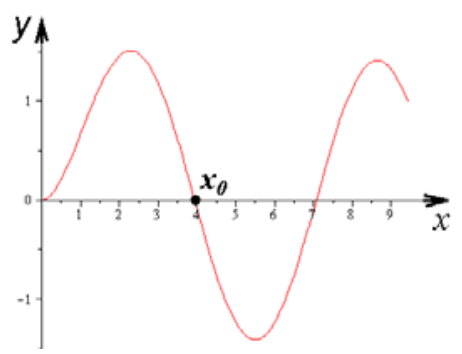

Fig. 2

In $D=\left\{(x, t) \in \mathbf{R}^{2}, x \in\left(0, x_{0}\right), t \in\left(0, t_{0}\right)\right\}$ let us consider an equation

$$
u_{t t t}-u_{t t}+u_{t}+u_{x x x x x x}-u_{x x x x}-u_{x x}+u=0
$$

with boundary conditions

$$
\begin{aligned}
& \left.u\right|_{t=0}=\left.u_{t}\right|_{t=0, t=t_{0}}=\left.u\right|_{x=0, x=x_{0}}=\left.u_{x}\right|_{x=0}=\left.u_{x x x x}\right|_{x=0, x=x_{0}}= \\
& =\left.u_{x x x x x}\right|_{x=0}=0 .
\end{aligned}
$$

A nonzero solution of this problem is the function

$$
\begin{aligned}
& u(x, t)=\left(e^{-x}+\sqrt{2} \sin \left(x-\frac{\pi}{4}\right)\right)\left(\sqrt{3}+e^{\frac{t}{2}}\left(\sin \left(\frac{\sqrt{3}}{2}\right) t-\right.\right. \\
& \left.\left.-\sqrt{3} \cos \left(\frac{\sqrt{3}}{2}\right) t\right)\right)=y_{1}(x) \cdot y_{2}(t) .
\end{aligned}
$$

Figures of graphs $y_{1}=y_{1}(x)$ (Fig. 3), $y_{2}=y_{2}(t)$ (Fig. 4), $u(x, t)=y_{1}(x) \cdot y_{2}(t)$ (Fig. 5). 


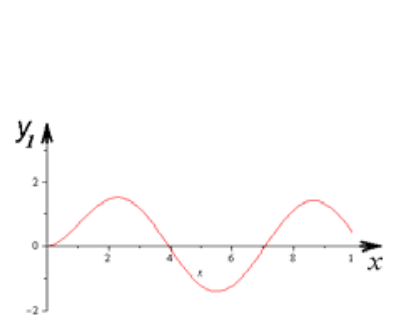

Fig. 3

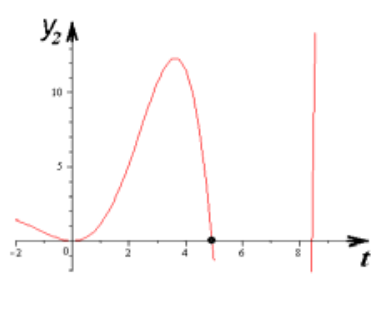

Fig. 4

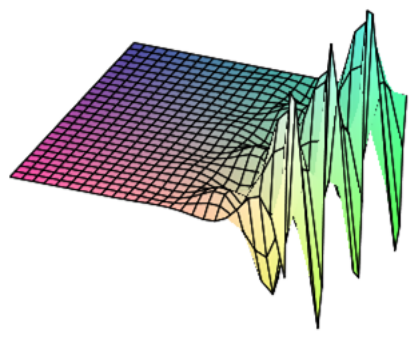

Fig. 5

\section{Second order differential equation (1)} with respect to $t$

In N.A. Chuesheva's and E.V. Maksimova's article [7] the following equation in $D=\{(x, t): x \in(0,1)$, $t \in(0,10\}$ was considered

$$
u_{t t}+e u_{t}-u_{x x x x}+a u_{x x x}+b u_{x x}+c u_{x}+d u=f(x, t)
$$

with boundary conditions

$$
\left.u\right|_{t=o, t=1}=\left.u\right|_{x=o, x=1}=\left.u_{x}\right|_{x=o, x=1}=0 .
$$

Let the space $H^{4,2}(D)$ be obtained by a closure of the space of functions $\mathbf{C}^{4}(\bar{D})$ satisfying boundary conditions (7) with respect to the norm

$$
\|u\|_{H^{4,2}(D)}=\left(\int_{D}\left(u^{2}+u_{x}^{2}+u_{t}^{2}+u_{x x}^{2}+u_{t t}^{2}+u_{x x x}^{2}+u_{x x t}^{2}+u_{x x x x}^{2}\right) d D\right)^{\frac{1}{2}} .
$$

Theorem 3. Let it be that $f(x, t) \in L_{2}(D)$. Let the following conditions be satisfied for coefficients of the Eq. (6): 1) $a \geq 0$; 2) $b>0$; 3) $\frac{\pi^{2}}{128}-d \geq \delta>0$. Then a solution $u(x, t)$ of the boundary problem (7) of the Eq. (6) from the space $H^{4,2}(D)$ exists and is unique.

A proof of the theorem is given in [7].

If we consider the following conditions for the Eq. (6) on boundary domain $D$

$$
\left.u\right|_{t=o, t=1}=\left.u\right|_{x=o, x=1}=\left.u_{x x}\right|_{x=o, x=1}=0
$$

then it is true that

Theorem 4. Let it be that $f(x, t) \in L_{2}(D)$, and coefficients of the Eq. (6) satisfy conditions: 1) $a \geq 0$; 2) $b>0$; 3) $(b-\varepsilon) \cdot \frac{\pi}{8}-d \geq \delta>0$. Then a solution $u(x, t)$ of the boundary problem (7) for the Eq. (6) from the space $H^{2,1}(D)$ exists is unique.
Here a norm in the space $H^{2,1}(D)$ is given by an equality

$$
\|u\|_{H^{2,1}(D)}=\left(\int_{D}\left(u^{2}+u_{x}^{2}+u_{t}^{2}+u_{x x}^{2}\right) d D\right)^{\frac{1}{2}} .
$$

The theorem is proved as in [7].

Problem 7. In $D=\left\{(x, t) \in \mathbf{R}^{2}, \quad x \in(0, \pi)\right.$, $t \in(0, \pi)\}$ with $a=4, b=3, c=4, d=5, e=0$ for the Eq. (6) with boundary conditions

$$
\left.u\right|_{t=o, t=\pi}=\left.u\right|_{x=o, x=\pi}=\left.u_{x x}\right|_{x=o, x=\pi}=0
$$

a function $u(x, t)=\sin x \cdot \sin t \neq 0$ is not a unique solution.

Problem 8. In $D=\left\{(x, t) \in \mathbf{R}^{2}, \quad x \in\left(0, \frac{2 \pi}{\sqrt{3}}\right)\right.$, $(t \in 0, \pi)\}$ let us consider an equation

$$
u_{t t}(x, t)+u_{x x x x}(x, t)+u_{x x x}(x, t)-u_{x}(x, t)=0
$$

with boundary conditions

$$
\left.u\right|_{t=0, t=\pi}=\left.u\right|_{x=0, x=\frac{2 \pi}{\sqrt{3}}}=\left.u_{x x x}\right|_{x=0, x=\frac{2 \pi}{\sqrt{3}}}=0 .
$$

The solution of problems (8) and (9) is a function

$$
u(x, t)=\sin t \cdot e^{-\frac{x}{2}} \sin \frac{\sqrt{3}}{2} x .
$$

Note. A derivative of any order with respect to a variable $x$ or $t$ of this function is not also a unique solutions of the problem (8) in a rectangle $D=\left\{(x, t) \in \mathbf{R}^{2}, x \in\left(x_{1}, x_{2}\right), t \in\left(t_{1}, t_{2}\right)\right\}$. For example, $u_{x}(x, t)=-\frac{1}{2} \sin t \cdot e^{-\frac{x}{2}}\left(\sin \frac{\sqrt{3}}{2} x-\sqrt{3} \cos \frac{\sqrt{3}}{2} x\right)=y_{1}(t) \cdot y(x)$ is a solution of (8) with boundary conditions

$$
\left.\left(u_{x}\right)\right|_{t=0, t=\pi}=\left.\left(u_{x}\right)\right|_{x=x_{1}, x=x_{2}}=\left.\left(u_{x}\right)_{x x x}\right|_{x=x_{1}, x=x_{2}}=0 .
$$

Fig. 6 represents a graph of the function $y(x)=-\frac{1}{2} e^{-\frac{x}{2}}\left(\sin \frac{\sqrt{3}}{2} x-\sqrt{3} \cos \frac{\sqrt{3}}{2} x\right)$.

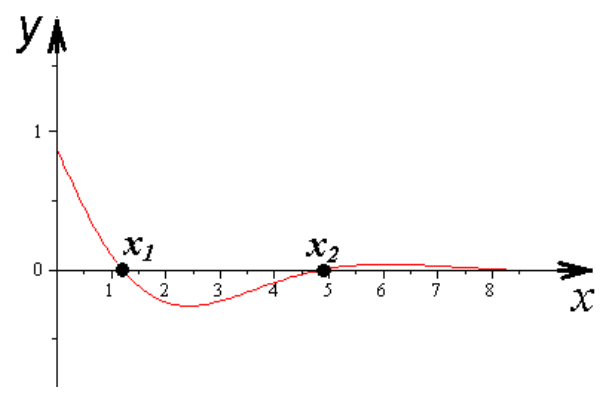

Fig. 6

Problem 9. In $D=\{(x, t): x \in(0, \pi), t \in(0,1)\}$ let us consider an equation 


$$
\begin{aligned}
& (1-t)^{2} u_{t t}-u_{x x x x}+a(x, t) u_{x x x}-u_{x x}+a(x, t) u_{x}+ \\
& +\frac{3}{4} u+(1-t) u_{t}=\frac{3}{4}\left(1-\frac{t}{2}\right) \sin x
\end{aligned}
$$

with boundary conditions

$$
\left.u\right|_{t=o}=\left.u_{t}\right|_{t=o}=\left.u\right|_{x=o, x=\pi}=\left.u_{x x}\right|_{x=o, x=\pi}=0 .
$$

With an analytical coefficient $a(x, t)$ and analytical right side in $\bar{D}$ of the Eq. (10) a solution of the problem

$$
u(x, t)=\left((1-t)^{\frac{3}{2}}-\left(1-\frac{3}{2} t\right)\right) \cdot \sin x
$$

doesn't belong to the class of functions $W_{2}^{2}(D)$ as in [1].

Problem 10. In $D=\{(x, t): x \in(0, \pi), t \in(0, \infty)\}$ let us consider an equation

$$
\left(u^{n}\right)_{t t}-\left(u^{n}\right)_{x x x x}+\left(u^{n}\right)_{x x}+\left(u^{n}\right)_{t}=0
$$

with boundary conditions

$$
\begin{aligned}
& \left.u^{n}\right|_{t=o}=\frac{\sin n x}{n^{5}},\left.\left(u^{n}\right)_{t}\right|_{t=o}=\frac{\sin n x}{n^{3}},\left.u^{n}\right|_{x=o, x=\pi}= \\
& =\left.\left(u^{n}\right)_{x x}\right|_{x=o, x=\pi}=0 .
\end{aligned}
$$

The unstable solution of this problem is a function $u(x, t)=\frac{e^{n^{2} t} \cdot \sin n x}{n^{5}}$.

\section{First-order differential equation (1) with respect to a derivative $t$}

In N.A. Chuesheva's and I.O. Korkina's article [8] in $D=\{(x, t): x \in(0,1), t \in(0,1)\}$ was considered an equation

$$
\begin{aligned}
& L u=k(t) u_{t}+u_{x x x x}+c(x, t) u_{x x x}+b(x, t) u_{x x}+ \\
& +a(x, t) u_{x}+d(x, t) u=f(x, t)
\end{aligned}
$$

with initial boundary conditions

$$
\left.u\right|_{t=0}=0,\left.u\right|_{\substack{x=0 \\ x=1}}=0,\left.u_{x}\right|_{\substack{x=0 \\ x=1}}=0 .
$$

Theorem 5. Let the right side of the Eq. (11) be a function $f(x, t) \in L_{2}(D)$. Let coefficients of the Eq. (11) satisfy conditions:

1) $\quad a(x, t), b(x, t), c(x, t), d(x, t) \in \quad \mathbf{C}^{4}(\bar{D})$, $k(t) \in \mathbf{C}^{3}[0,1]$;

2) $c(0, t) \leq 0, c(1, t) \leq 0, c_{x}(x, t)<0,(x, t) \in \bar{D}$;

3) $k(1) \leq 0$;

4) let there be real constants $\lambda, \delta_{1}, \delta_{2}$ such that

$$
\begin{aligned}
& -\lambda \cdot k(t)-k_{t}(t)-a_{x}(x, t)+b_{x x}(x, t)-c_{x x x}(x, t)+ \\
& +2 d(x, t) \geq \delta_{1}>0, \quad(x, t) \in \bar{D}, \\
& -2 b(x, t)+3 c_{x}(x, t) \geq \delta_{2}>0, \quad(x, t) \in \bar{D} .
\end{aligned}
$$

Then there exists and is single a solution $u(x, t)$ of the boundary problem (12) for the Eq. (11) from the space $H^{4,1}(D)$.

$H^{4,1}(D)$ is a Hilbert space with the norm

$$
\|u\|_{H^{4,1}(D)}=\left(\int_{D}\left(u^{2}+u_{x}^{2}+u_{t}^{2}+u_{x x}^{2}+u_{x x x}^{2}+u_{x x x x}^{2}\right) d D\right)^{\frac{1}{2}} .
$$

Theorem is proved in [8].

Note. Let coefficients of the Eq. (11) be, for example, the following functions

$$
\begin{gathered}
a(x, t)=x+1 ; d(x, t)=5 ; \quad c(x, t)=-2 x ; \\
b(x, t)=-x-8 ; \quad k(t)=-t^{2} .
\end{gathered}
$$

Constants, for example, are $\lambda=1, \delta_{1}=8, \delta_{2}=9$. Then all theorem conditions are satisfied.

Problem 11. If we set another conditions for the Eq. (11) on the boundary of $D$

$$
\left.u\right|_{t=0}=0,\left.u_{x}\right|_{\substack{x=0 \\ x=1}}=0,\left.u_{x x x}\right|_{\substack{x=0 \\ x=1}}=0,
$$

then we can prove the following theorem as the theorem 5

Theorem 6. Let the right side of the Eq. (11) be a function $f(x, t) \in L_{2}(D)$. Let coefficients of the Eq. (11) satisfy conditions: 1) $a(x, t), b(x, t), c(x, t)$, $d(x, t) \in \mathbf{C}^{4}(\bar{D}), \quad k(t) \in \mathbf{C}^{3}[0,1]$

Let the following conditions be satisfied for these coefficients in $\bar{D}$ :

1) $k(1) \leq 0$;

2) $a(0, t)-b_{x}(0, t)+c_{x x}(0, t) \geq 0, a(1, t)-b_{x}(1, t)+$ $+c_{x x}(1, t) \geq 0, t \in[0,1]$;

3) let there be real constants $\lambda, \delta_{1}, \delta_{2}, \delta_{3}$ such that

$$
\begin{aligned}
& -\lambda \cdot k(t)-k_{t}(t)-a_{x}(x, t)+b_{x x}(x, t)-c_{x x x}(x, t)+ \\
& +2 d(x, t) \geq \delta_{1}>0, \\
& \quad-2 b(x, t)+3 c_{x}(x, t) \geq \delta_{2}>0, \quad(x, t) \in \bar{D}, \\
& \left(\frac{8}{\pi}(1-\delta)-\frac{1}{2} \max _{(x, t) \in D}\left|c_{x}(x, t)\right|\right) \geq \delta_{3}>0, \quad 0<\delta<0,1 .
\end{aligned}
$$

Then a solution $u(x, t)$ of the boundary problem (13) for the Eq. (11) from the space $H^{4,1}(D)$ exists and is unique.

Note. For example, let coefficients of the Eq. (11) be the following functions

$$
\begin{gathered}
a(x, t)=x+1 ; d(x, t)=5 ; \quad c(x, t)=-2 x ; \\
b(x, t)=-x-8 ; \quad k(t)=-t^{2} .
\end{gathered}
$$

Constants, for example, are $\lambda=1, \delta_{1}=8$, $\delta_{2}=9, \delta_{3}=0,5$. Then all theorem conditions are satisfied.

Problem 12. In $D=\{(x, t): x \in(0, \pi), t \in(0,1)\}$ let us consider a model equation

$$
(1-t) u_{t}+u_{x x x x}+a(x, t) u_{x x x}-u_{x x}+a(x, t) u_{x}-\frac{3}{2} u=-\frac{1}{2} \sin x
$$


with initial condition $\left.u\right|_{t=0}=0$ and boundary conditions $\left.u_{x}\right|_{\substack{x=0 \\ x=\pi}}=0 ;\left.u_{x x x}\right|_{\substack{x=0 \\ x=\pi}}=0$.

Functions $a(x, t),(1-t),-\frac{1}{2} \sin x$ are analytical in $D$. Although a solution of the problem is a function

$$
u(x, t)=(\sqrt{1-t}-1) \cos x \notin H^{4,1}(D) .
$$

Problem 13. Consider the Korteweg-de Vries linearized equation

$$
u_{t}(x, t)+u_{x x x}(x, t)=0 .
$$

A solution of this equation is a complex function with $c \neq \pm d$

$$
u(x, t)=b e^{-a t} \cdot\left(c e^{-\frac{1}{2} \sqrt[3]{a}(1+i \sqrt{3}) x}+d e^{-\frac{1}{2} \sqrt[3]{a}(1-i \sqrt{3}) x}+l e^{\sqrt[3]{a} x}\right)
$$

A real solution of this equation is a function

$$
u(x, t)=b e^{-t} \cdot e^{-\frac{x}{2}}\left(\sin \frac{\sqrt{3}}{2} x+\sqrt{3} \cos \frac{\sqrt{3}}{2} x\right) .
$$

Note, that (14) is a solution for the following equation with continuous coefficients

$$
\begin{aligned}
& a(x, t) u_{t}(x, t)+b(x, t) u_{x x x x x}(x, t)+c(x, t) u_{x x x x}(x, t)+ \\
& +a(x, t) u_{x x x}(x, t)-b(x, t) u_{x x}(x, t)-c(x, t) u_{x}(x, t)=0
\end{aligned}
$$

with conditions on the boundary of

$$
\begin{array}{r}
D=\left\{(x, t): x \in\left(0, \frac{2 \pi}{3}\right), t \in(0, \infty)\right\} \\
\left.u\right|_{x=0, x=\frac{2 \pi}{3}}=\left.u_{x x x}\right|_{x=0, x=\frac{2 \pi}{3}}=0 .
\end{array}
$$

\section{Some exact solutions of Korteweg-de Vries, Degasperis-Procesi and Ramani equations.}

In N.A. Chuesheva's papers [8] and [9] exact solutions for the Korteweg-de Vries, BurgersKorteweg-deVries, and Kadomtsev-Petviashvili nonlinear equations are written out.

Problem 14. In [10] the Cauchy problem for the following fifth-order Korteweg-de Vries equation is considered

$$
\begin{gathered}
u_{t}-u_{x x x x x}+c_{1}\left(u^{3}\right)_{x}+c_{2}\left(\left(u_{x}\right)^{2}\right)_{x}+c_{3}\left(u u_{x x}\right)_{x}=0 \mathbf{R}^{2}, \\
u(0, x)=u(x), x \in \mathbf{R},
\end{gathered}
$$

where $c_{1}, c_{2}, c_{3} \in \mathbf{R}, c_{3} \neq 0$. A function $u(x, t)$ is real-valued or complex. Conditions of problem correctness are written out in (10).

In this article let us write out some particular solutions of this equation if:

1) $c_{1}=c_{2}=c_{3}=1$ then there is a real function

$$
\begin{gathered}
u(x, t)=-\frac{4 b^{2}}{3} \frac{(65+\sqrt{145})}{3-\sqrt{145}}+ \\
+\frac{2 b^{2}(25-31 \sqrt{145})}{77-3 \sqrt{145}} \tanh ^{2}\left(-a-b x+2 b^{5}(17+\sqrt{145}) t\right) ;
\end{gathered}
$$

2) $c_{1}=-1, c_{2}=c_{3}=1$ then there is a complex function

$$
\begin{gathered}
f(z)=-\frac{4 b^{2}}{3} \frac{(55+i \sqrt{95})}{3+i \sqrt{95}}- \\
-\frac{2 b^{2}(35+29 i \sqrt{95})}{43-3 i \sqrt{95}} \tanh ^{2}\left(-a-b x+2 b^{5}(7+i \sqrt{95}) t\right)
\end{gathered}
$$

3) $c_{2}=-1, c_{1}=c_{3}=1$ then there is a real function $u(x, t)=8 b^{2}-12 b^{2} \tanh ^{2}\left(-a-b x+16 b^{5} t\right)$.

For example, if $b=1, \quad y=-x+16 t$, $a=\frac{1}{2} \cdot \ln \left(\frac{1+\frac{\sqrt{2}}{\sqrt{3}}}{1-\frac{\sqrt{2}}{\sqrt{3}}}\right)$ a function graph $4 z=u(y)=$ $\left.=8-12 \tanh ^{2}(-a+y)\right)$ takes the form (Fig. 7).

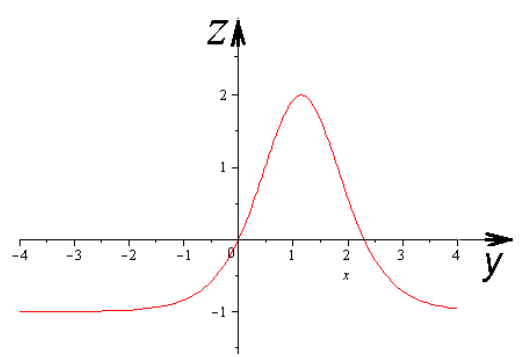

Fig. 7

Problem 15. In [11] the Cauchy problem and local correctness for the Degasperis-Procesi equation with the following variance term are studied

$$
u_{t}+u_{t x x}+u_{x x x}-u_{x x x x x}+4 u u_{x}=3 u_{x} u_{x x}+u u_{x x x}, u(x, 0)=u_{0} .
$$

Bourgain's method which refers to the Kortewegde Vries periodic equation and Schrödinger equation is used to prove existence of the Cauchy problem solution for this equation. Let us write out exact solution of this equation

$$
u(x, t)=8 b^{2}-\frac{3}{5}-12 b^{2} \tanh ^{2}\left(-a+b x+\frac{12}{5} b t\right) .
$$

For example, if $b=1, \quad y=x+\frac{12}{5} t$, $a=\frac{1}{2} \cdot \ln \left(\frac{1+\sqrt{\frac{37}{60}}}{1-\sqrt{\frac{37}{60}}}\right)$ then $u(x, t)$ a function graph takes the form as for $z=u(y)=2-3 \tanh ^{2}(-a+y)$.

Problem 16. In [12] the following nonlinear differential Ramani equation of the sixth order was considered

$$
\begin{aligned}
& u_{x x x x x x}+15 u_{x} u_{x x x x}+15 u_{x x} u_{x x x}+45\left(u_{x}\right)^{2} u_{x x}-5 u_{x x x t}- \\
& -15 u_{x} u_{x t}-15 u_{t} u_{x x}-5 u_{t t}=0, u=u(x, t) .
\end{aligned}
$$

With the help of $G^{\prime} / G$-method a solution of this differential equation was found. 
Exact solution of the Ramani equation is given in [13]. Let us consider a similar fourth-order equation

$a u_{x}(x, t) u_{x x x x}(x, t)+b u_{x x}(x, t) u_{x x x}(x, t)+c\left(u_{x}(x, t)\right)^{2} u_{x x}(x, t)+$ $+d u_{x}(x, t) u_{x t}(x, t)+e u_{t}(x, t) u_{x x}(x, t)=0$,

$a, b, c, d, e$ are real constants and $c \neq 0, d+e \neq 0$. The function

$u(x, t)=c_{3}-\frac{6 c_{2}(b+2 a) \tanh \left(-c_{1}-c_{2} x+\frac{4 c_{2}^{3}(a+b) t}{d+e}\right)}{c}$

is a solution of this equation.

In a bounded domain $D$ with a smooth boundary $\Gamma$ a function

$$
u(x, y)=a+2 \sqrt{2} \tanh \left(-b+\frac{\sqrt{2}}{2}(x+y)\right)
$$

is a solution of the fourth-order nonlinear equation

$$
\begin{aligned}
& u_{x} \cdot u_{y y x x}-u_{x x x}+u_{y y y}-u_{x x y}+u_{x y y}+3\left(u_{x}\right)^{2} \cdot u_{x x}- \\
& -u_{x} \cdot u_{x y}-u_{y} u_{x x}=0 .
\end{aligned}
$$

Problem 17. In two-dimensional geometry the following Kadomtsev-Petviashvili equation is a generalization of the third-order Korteweg-de Vries equation:

$$
\frac{\partial}{\partial x}\left(u_{t}+u_{x x x}+6 u u_{x}\right)-a u_{y y}=0 .
$$

In [9] the exact solution of this equation is written out

$$
\begin{aligned}
& 6 \cdot c_{2}^{2} \cdot u(x, t, y)=\left(-c_{3} c_{2}+a c_{4}^{2}+8 c_{2}^{4}-\right. \\
& \left.-12 c_{2}^{4} \tanh ^{2}\left(c_{1}+c_{2} x+c_{3} t+c_{4} y\right)\right) .
\end{aligned}
$$

A function graph is the same as for $z=u(y)=2-3 \tanh ^{2}(-a+y)$.

Problem 18. If any dissipation, the third-order Korteweg-de Vries equation goes over into the Bürgers-Korteweg-de Vries equation

$$
u_{t}+u_{x x x}+6 u u_{x}-a u_{x x}=0 .
$$

In [9] the exact solution of this equation is written out

$$
u(x, t)=\frac{3 a^{3}-250 c_{3}}{150 a}-
$$

$$
\begin{aligned}
& -\frac{a^{2}}{25} \tanh \left(c_{1}+\frac{1}{10} a x+c_{3} t\right)-\frac{a^{2}}{50} \tanh ^{2}\left(c_{1}+\frac{1}{10} a x+c_{3} t\right) . \\
& \text { Let } a=1, c_{3}=\frac{4}{250}, y=c_{1}+\frac{1}{10} a x+c_{3} t \text { then } a
\end{aligned}
$$

function graph

$z=150 \cdot u(y)$ take the form shown on Fig. 8.

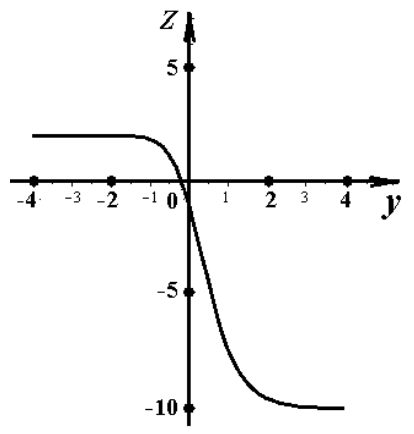

Fig. 8

\section{CONCLUSION}

For the first time we have constructed new examples of ill-posed formulations of boundary problems for some linear and nonlinear partial differential equations of the higher order that are connected with some important modern equations of mathematical physics. In particular, we have constructed examples and graphs of explicit solutions for the third and fifth-order Korteweg-de Vries equations, linearized Korteweg-de Vries equation, Degasperis-Procesi equation and Ramani equation. Also we have given a review on results obtained by other authors.

\section{REFERENCES}

1. Vragov V.N. Kraevye zadachi dlya neklassicheskikh uravneniy matematicheskoy fiziki [Boundary value problems for nonclassical equations of mathematical physics]. Novosibirsk: Novosibirsk University, 1983. 90 p.

2. Demidenko G.V., Uspenskii S.V. Uravneniya i sistemy, ne razreshennye otnositel'no starshey proizvodnoy [Equations and systems are not permitted with respect to the highest derivative]. Novosibirsk Scientific Book, 1998. 438 p.

3. Egorov I. E., Pyatkov S. G., Popov S.V. Neklassicheskie differentsial'no-operatornye uravneniya [Nonclassical differentialoperator equations]. Novosibirsk: Publishing House of Science, 2000. 336 p.

4. Kozhanov A.I. Nachal'no-kraevaya zadacha dlya uravneniya tipa obobshchennogo uravneniya Busineska s nelineynym istochnikom [Initial boundary value problem for the generalized Boussinesq equation type with a nonlinear source]. Mat. Deputy, 1999, vol. 65, no. 1, pp. 70-75.

5. Chueshev A.V. Ob odnom nelineynom uravnenii smeshannogo tipa nechetnogo poryadka [On a nonlinear equation of mixed type of odd order]. Vestnik Novosib. un-ta, seriya "matematika, mekhanika, informatika" [Bulletin Novosib. University, series "Mathematics, Mechanics and Computer Science"]. Novosibirsk, 2001, vol. 1, no. 1, pp. 107-123.

6. Chuesheva N. A. Ob odnoy kraevoy zadache dlya nelineynogo uravneniya tret'ego poryadka [On a boundary value problem for nonlinear third-order equations]. Differentsial'nye uravneniya [Differential Equations]. Minsk, 1980, vol. 16, no. 1, pp. 183-185.

7. Chuesheva N. A., Maksimova E.V. Kraevaya zadacha dlya differentsial'nogo uravneniya chetvertogo poryadka [Boundary value problem for the fourth-order differential equation]. Vestnik KemGU [Bulletin of Kemerovo State University]. Kemerovo, 2011, no. 3/1, pp. 266-268.

8. Chuesheva N. A. Neskol'ko lineynykh i nelineynykh differentsial'nykh uravneniy vysokogo poryadka [Some linear and nonlinear differential equations of higher order]. Dni geometrii v Novosibirske tez. mezhdunar. konf. Novosibirsk, 26-29 avgusta [Days of geometry in Novosibirsk: mes. Intern. Conf.], Novosibirsk, 26-29 of August. Novosibirsk Institute of 
Mathematics named after S.B. Sobolev. Novosibirsk, 2015, pp. 67-68.

9. Chuesheva N. A. Kraevye zadachi dlya nekotorykh uravneniy tret'ego poryadka [Boundary problems for some of the thirdorder differential equations]. Dni geometrii v Novosibirske: tez. mezhdunar. konf., Novosibirsk, 24-27 sentyabrya [Days of geometry in Novosibirsk: mes. Intern. Conf., Novosibirsk, 24-27 of September.] Novosibirsk Institute of Mathematics named after S.B. Sobolev. Novosibirsk, 2014, pp. 80-81.

10. Takamori Kato. Well-posedness for the fifth order KdV equation. Funkc. Ekvacioj=Funct. Equat., 2012, vol. 55, no. 1, pp. 17-53.

11. Shu Wen. The Cauchy equation of the Degaspersive - Procesi equation with the dispersive term. Nonlinear Sci, 2008, vol. 5, no. 3, pp. 210-216.

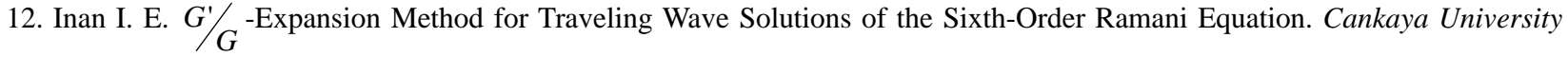
Journal of Science and Engineering, 2010, vol. 7, no. 1, pp. 51-57.

13. Chuesheva N.A. Neskol'ko lineynykh i nelineynykh differentsial'nykh uravneniy [Some linear and nonlinear differential equations]. Dni geometrii v Novosibirske: tez. mezhdunar. konf., Novosibirsk, 28-31 avgusta [Days of geometry in Novosibirsk: mes. Intern. Conf., Novosibirsk, 28-31 of August]. Novosibirsk, Novosibirsk Institute of Mathematics named after S.B. Sobolev, 2013, pp. 93-94.

\section{Nadezda A. Chuesheva}

Cand.Sci.(Phys.-Math.), Associate Professor, Associate Professor of the Department of Fundamental Mathematics, Kemerovo State University, Kemerovo, Russian Federation.

\section{Aleksandr V. Chueshev}

Cand.Sci.(Phys.-Math.), Associate Professor, Associate Professor of the Department of Fundamental Mathematics, Kemerovo State University, Kemerovo, Russian Federation. 